\title{
Ear Dimple
}

National Cancer Institute

\section{Source}

National Cancer Institute. Ear Dimple. NCI Thesaurus. Code C87071.

A pit or dimple in front of the ear of a newborn is a common finding; rarely, such a dimple may get infected, which will be manifested with redness and swelling and will require medical attention. 